\title{
Alexander Parshin (March 13, 1939-April 2, 2013)
}

Published online: 29 June 2013

(C) Springer Science+Business Media New York 2013

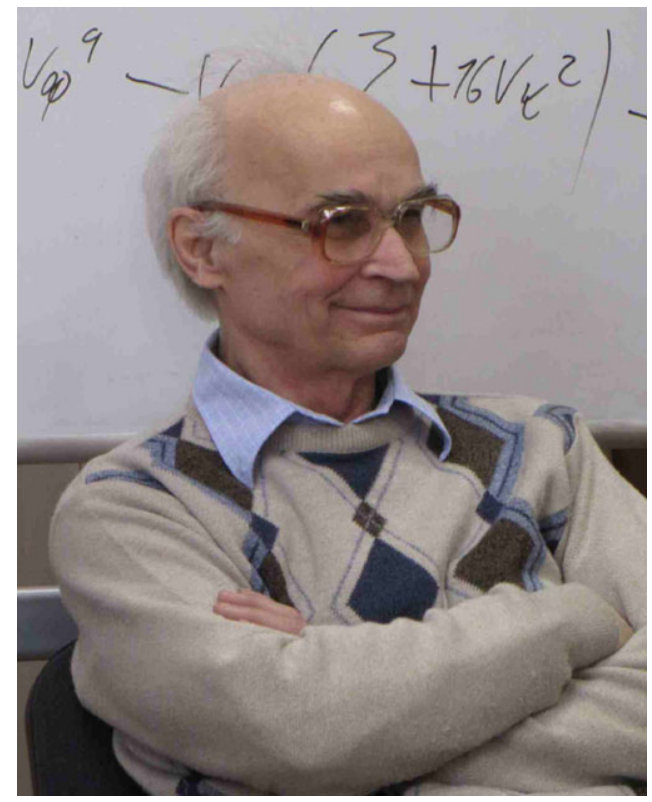

The Editors of the Journal of Low Temperature Physics regret to inform you about the death of Academician Alexander Parshin who was a member of our Editorial Board. Among the variety of Alexander Parshin's discoveries in low temperature physics, the most important one is probably that on the crystallization waves in solid and superfluid ${ }^{4} \mathrm{He}$ that he predicted to exist in 1978 and observed experimentally in 1979 with his colleagues in the P.L. Kapitza Institute in Moscow. His main scientific interest was the physics of quantum surfaces such as the surface of helium superfluids and the solid-liquid interface in helium, in which field his contributions cannot indeed be overestimated. We extend our condolences to his family, friends, co-workers, students, and to everyone who knew Alexander Parshin. 